\title{
Delay between Discharge and Admit Time Delay in ADT-A03 messages via LEEDS
}

\author{
Jose A. Serrano* \\ Office of Public Health, Louisiana Department of Health, New Orleans, LA, USA
}

\section{Objective}

To explore the difference between the reported date of admission and discharge date using discharge messages (A03), from hospital emergency departments participating in the Louisiana Early Event Detection System (LEEDS.

\section{Introduction}

The Infectious Disease Epidemiology Section (IDEpi) within the Office of Public Health (LaOPH) conducts syndromic surveillance of emergency departments by means of the Louisiana Early Event Detection System (LEEDS). LEEDS accepts ADT (admit-dischargetransfer) messages from participating hospitals, predominately A04 (registration) and A03 (discharge), to obtain symptom or syndrome information on patients reporting to hospital emergency departments. Capturing the data using discharge messages (A03) only could result in a delay in receipt of data by $\mathrm{LaOPH}$, considering the variability in the length of stay of a patient in the ED.

\section{Methods}

Emergency department data from participating hospitals is imported daily to LEEDS and processed for syndrome classification. IDEpi syndromic surveillance messages received for the period of CDC week 1632 and 1636 (8/8/16-9/8/16) using MS Access and Excel to calculate the difference (in days) between the reported admit date and discharge date in A03 messages.

\section{Results}

$88.1 \%$ of the A03 messages submitted in the 4 week analysis period exhibited no delay (delay $=0$ days) between the admit date and the reported discharge date, compared to only $10.7 \%$ showing a delay of one day (delay = 1 day) and $1.06 \%$ showing a delay of 2 days or more (delay $\geq 2$ days). Less than $0.2 \%$ of the messages had missing information regarding discharge date (Table 1).

\section{Conclusions}

Syndromic surveillance systems operate under a constant need for improvement and enhancement. The quality of the data, independent of the quality of the system, should always strive to be of the highest pedigree in order to inform disease-specific programs and detect public health aberrations. In order to identify these potential concerns, it is imperative that the data be submitted to public health agencies in a timely manner. Based on this analysis, the lapse in time between admit and discharge results in little to no patient syndromic data delay for those hospital ED's that exclusively send A03 messages. This statement is supported by the finding that close to $99 \%$ of messages demonstrated a delay between admit date and discharge date of one day or less.

Table 1. Delay between reported Admit and Discharge date in A03 messages submitted to LEEDS

\begin{tabular}{|c|c|c|}
\hline & Number & $\%$ \\
\hline Missing Discharge Date & 75 & 0.12 \\
\hline Delay $=0$ days & 56753 & 88.08 \\
\hline Delay $=1$ day & 6922 & 10.74 \\
\hline Delay $>=2$ days & 683 & 1.06 \\
\hline Total & 64433 & 100 \\
\hline
\end{tabular}

Keywords

Syndromic; Surveillance; Quality

\section{*Jose A. Serrano}

E-mail: jose.serrano@la.gov 